diagnosis is now Kikuchi lymphadenitis. Unfortunately, he was presented with fever and low WBC count again in September 2014 and went to NUH Singapore and his current working diagnosis is recurrent Kikuchi lymphadenitis with Mycoplasma and Infectious mononucleosis infection. His PET CT scan reported as increased mediastinal masses with low significance. Currently he is under care of Palliative medicine of RIPAS hospital.

Discussion Our case illustrates a significant role of palliative medicine for the complicated clinical case. Which could be achieved by meticulous history taking and symptoms assessment and relevant investigations. Hence, integrated palliative care service could prevent the miss diagnosis, and ensures a better outcome.

\section{P-71 EVALUATION OF A RAPID DISCHARGE PATHWAY FOR DYING PATIENTS}

Sally Hall. Royal Surrey County Hospital, Guildford, UK

10.1136/bmjspcare-2017-00133.71

Background The rapid discharge pathway for the dying patient is an integral part of the Department of Health's End of Life Care Strategy. It is aimed at patients who are thought to be in the last 72 hours of life, although there is no clear guidance about identifying such patients.

The aim of this prospective service evaluation was to review the outcomes of patients started on the rapid discharge pathway for the dying patient.

Methods Data was prospectively collected on all patients started on the rapid discharge pathway during a six month period (February - July 2016) at a medium sized district general hospital with associated cancer centre. Data collected included: date of referral for rapid discharge; date of discharge; date of death; place of death; readmissions; demographic details.

Results 24 patients were referred for the rapid discharge pathway, 23 were thought to be appropriate for RDP (9 patients with non-malignant disease, 14 patients with cancer). 18 patients were discharged home, whilst 5 patients died in hospital (all within 72 hours). Of the 18 patients discharged 16 have died ( 7 within 72 hours, 9 after more than 72 hours). In total 17 out of 23 patients died within 72 hours. No patients were readmitted to hospital.

Conclusion The rapid discharge pathway can help facilitate discharge home for patients in the last days of life. However prognostication can be challenging, especially differentiating between patients who will die within 72 hours (more difficult) than patients who will die within 1 week (less difficult).

\section{P-72 'PLACE BONDING' IN CHILDREN'S HOSPICE CARE \\ 1,2Helena Dunbar. 'De Montfort University, Leicester, UK; ${ }^{2}$ Rainbows Hospice for Children} and Young People

\subsection{6/bmjspcare-2017-00133.72}

As the number of children in the UK with life-limiting and life-threatening conditions are increasing, providers of palliative care services, such as children's hospices are considering how best to support families. However, evidence suggests that only a small percentage of parents of children with such conditions choose to access hospice services.

This exploratory study examined parents' perspectives of existing hospice services, the types and characteristics of hospice services parents wanted, the barriers and facilitators to accessing services, and how best these services could be delivered to meet the needs of the population of children with life-limiting and life-threatening conditions in one region in England.

A two phase qualitative study underpinned by a constructivist grounded theory methodology was employed. In Phase 1 focus groups were used to collect data from twenty four parents of children accessing services at the hospice. In Phase 2 in-depth semi-structured interviews were conducted with seven parents of children who did not use services at the hospice and with a further seven parents who had either previous experience of the hospice or were using a hospice outside of the region.

Findings showed the desire, the sense of searching that parents had in seeking out a place, other than their actual home, where their child could access a caring environment and the parent/s receive some respite from caring. Over time affective bonds developed between parents and the hospice. Finding a place where they belonged and where they felt at 'home' made the decision to accept help in caring for their child with a life-limiting condition more manageable.

A theory of place bonding was developed which has the potential to influence practice for children's hospices and also provide a platform for service development for other respite situations for children and young people with life-limiting conditions.

\section{P-73 IMPROVING PALLIATIVE DEMENTIA CARE - EVALUATION OF A NEW EDUCATION PROGRAMME}

${ }^{1,2}$ Clare White, ${ }^{3}$ Clare McVeigh, ${ }^{1}$ Sue Foster, ${ }^{4}$ Lynn Dunwoody, ${ }^{1}$ Max Watson. ${ }^{1}$ Northern Ireland Hospice, Belfast, Northern Ireland; ${ }^{2}$ Belfast HSC Trust, Northern Ireland; ${ }^{3}$ Queens University Belfast, Northern Ireland; ${ }^{4}$ Ulster University, Northern Ireland

\subsection{6/bmjspcare-2017-00133.73}

Background Globally, dementia is a growing healthcare problem. Both the life-limiting nature of the illness and the clinical manifestations of the disease warrant patients and their families being able to access a palliative approach to care. Due to the complex symptomatology associated with dementia, providing optimal holistic care can be challenging and healthcare professionals (HCPs) need the appropriate level of knowledge and skills in both palliative and dementia care. The European Certificate in Holistic Dementia Care (ECHDC) is an 8 week, multidisciplinary home study programme designed to help meet this need. The course was created by palliative and dementia specialists. The aim of this research was to evaluate the effectiveness of the course in improving the knowledge and skills of HCPs regarding a palliative approach to dementia care.

Methods A prospective mixed methods longitudinal cohort study was conducted to evaluate the education program. The first phase incorporated baseline knowledge and self-efficacy assessments prior to commencement of the course. The second phase encompassed post course knowledge and self-efficacy assessments, and focus groups exploring participants' experiences and perceptions of the course in enhancing their knowledge and skills in relation to palliative dementia care. 